\title{
Modeling Cognitive Activity of the Human Brain by the Mathematical Apparatus of Quantum Mechanics
}

\author{
Alexandr Petukhov, ${ }^{1, *}$ \\ ${ }^{1}$ Keldysh Institute of Applied Mathematics RAS, RU-125047, Moscow, Russia
}

\begin{abstract}
This paper discusses the pos sible approaches to modeling the cognitive activity of the human brain using the $m$ athematical a pparatus of qua ntum m echanics ( primarily - potential w ells a nd virtual particles) in terms of the theory of information representations. The article briefly describes the proposed theory of i nformation $r$ epresentations, draws an alogies, an $\mathrm{d} i$ dentifies co mmon $\mathrm{f}$ eatures o f i nformation representations of the human mind and Feynman's virtual particles. The human mind is represented as a one-dimensional potential well with finite walls of different sizes and internal potential barrier simulating the boundary between consciousness and subconsciousness. This creates a foundation for a mathematical apparatus that can make it possible to forecast particular cognitive functions of the human brain. The results of these studies can be used to create predictive models of various cognitive disorders (diseases) and to be used in diagnostics.
\end{abstract}

\section{Introduction}

Natural s cience as pects o $\mathrm{fh}$ ow $\mathrm{t}$ he e nvironmental information factors $\mathrm{i}$ mpact liv ing systems a re $\mathrm{s}$ till relatively p oorly studied [1]. $\mathrm{T}$ here $\mathrm{h}$ as been $\mathrm{q}$ uite a number of new e lectrophysiological methods c reated, primarily, the r egistration of e voked a nd e vent-related potentials recorded in experimental behavioral situations, that made $\mathrm{i} t$ pos sible $\mathrm{t}$ o a pproach $\mathrm{t}$ he $\mathrm{s}$ tudy of physiological $\mathrm{m}$ echanisms of individual stages o $\mathrm{f}$ information pr ocessing: sensory a nalysis, a ttention mobilization, i mage $f$ ormation, e xtraction of memory standards, $\mathrm{d}$ ecision making, et $\mathrm{c}$. The s tudy o $\mathrm{ft}$ ime parameters of electrophysiological reactions to stimuli of different $t$ ypes a nd $u$ nder $d$ ifferent $\mathrm{c}$ onditions made it possible for the first time to register time parameters, i.e. to estimate the duration of individual stages of information pr ocessing di rectly a $t$ the level of br ain substrate [ 1-2]. $\mathrm{M}$ any $\mathrm{f}$ undamental $\mathrm{f}$ acts were al so obtained u sing c lassical methods of ps ychology, which were used to study and analyze the behavior of subjects in various social situations.

The $\mathrm{p}$ roblem o $\mathrm{fd}$ escribing $\mathrm{t}$ he $\mathrm{p}$ rocesses o $\mathrm{f}$ information transfer and processing by a $\mathrm{n}$ individual is fundamental f or m odern cognitive s cience. Relatively recently, unique natural s cience models of i nformation transfer $\mathrm{f}$ rom in dividual to i ndividual [2-5], c ognitive activity modeling [ 6-13], an $\mathrm{d}$ others $\mathrm{h}$ ave ap peared. However, many o ft he $\mathrm{p}$ resented models ar e ei ther poorly scalable or insufficiently formalized and therefore do not allow to fundamentally explain the processes of information tr ansfer a nd its $d$ istortion $r$ esulting $f$ rom interaction with $t$ he external co mmunicative environment.
Moreover, one as pect of human co gnitive activity is the fact that a p erson's thinking is not c ode-based (as compared t o a co mputer), b ut i s a r esult o ft he interaction of multiple i mages. Although $t$ hese i mages have quite specific material structure at the heart of their functioning (namely, electrical and chemical a ctivity in the human brain), from the point of view of conventional mathematical models $t$ heir $d$ escription is $d$ ifficult for several reasons [11].

Therefore, $t$ his pa per pr oposes new methods $f$ or describing $t$ he act ivity o f i nformation $r$ epresentations (which, i $\mathrm{n} t$ urn, $\mathrm{s}$ imulate $\mathrm{t}$ he $\mathrm{c}$ ognitive a ctivity of a $\mathrm{n}$ individual) $b$ ased o $\mathrm{nt}$ he mathematical ap paratus o $\mathrm{f}$ quantum physics ( potential wells a nd $v$ irtual $p$ articles used i $\mathrm{n}$ phy sics $\mathrm{t}$ o de scribe $\mathrm{f}$ undamental i nteractions [14]).

\section{A brief overview of the theory of information representations}

The proposed theory is based on the idea of a universal cognitive unit [11] of information in the human mind an i nformation $r$ epresentation ( or i mage), t he space in which it exists, its topology and properties. Information representations ( hereinafter $r$ eferred $t o$ as I Rs) can b e defined as displays of objects and events in any feature space.

Correspondingly, $\mathrm{t}$ he $\mathrm{t}$ heory o $\mathrm{fi}$ nformation representations (hereinafter referred to as TIR) is a w ay to $\mathrm{d}$ escribe in formation interactions o $\mathrm{f}$ in dividuals, a $\mathrm{s}$ well as several cognitive functions of a person. 
TIR vi ews t he h uman mind a s a huge s tructured number o fi nteracting i mages, which ar e co nstantly affected by external factors.

Images with higher energy (we introduce the notion of en ergy $E$ to d escribe the communicative act ivity of images) are located "above" and closer to the edge of the individual's information image space, which is why they interact with e xternal e lements much more o ften; meanwhile, i mages with 1 ower e nergy and l onger response rate are located closer to the center of the space and $r$ elatively $r$ arely $i$ nteract act ively with e xternal stimuli.

TIR $\mathrm{g}$ ives an al ternative perspective o $\mathrm{n}$ some characteristic $r$ egularities in the h uman mind, a llowing for a co rrect interpretation a nd explanation of s ome of them.

The $\mathrm{i}$ nformation $\mathrm{i}$ mage can not b e co mmunicated between individuals without changes. Each IR is unique because each $\mathrm{i}$ ndividual $\mathrm{h}$ as specific $\mathrm{i}$ ndividual experiences.

Besides, an individual is unable to convey the image that $\mathrm{e}$ xists in $\mathrm{t}$ heir mind, in their I R s pace to a nother individual $\mathrm{d}$ irectly. $\mathrm{F}$ or th is purpose, th ey use $\mathrm{v}$ arious communication apparatuses formed within the social superstructure of communication or the communication field ( $C F)$. A c ommunication $f$ ield $(C F)$ is a $n$ information c ommunity o $\mathrm{f} i$ ndividual e xperiences a nd collective unconsciousness formed a s a r esult o f a $n$ individual's presence in society. The communicative apparatus includes speech, visual, tactile, s ymbolic, and other ways of information transmission.

In $\mathrm{p}$ revious works, th e mathematical model o $\mathrm{f}$ interaction of information representations was based on diffusion e quations ( first o fa $11, \mathrm{o} \mathrm{nt}$ he Langevin equation). $\mathrm{T}$ his ap proach al lowed $\mathrm{t}$ o $\mathrm{d}$ escribe s everal special cas es of co gnitive p rocesses but al so had s ome limitations related to the specificity of IRs. More details about TIR and its application to real cognitive processes and experimental testing are available in [15].

\section{A brief overview of the theory of information images}

What is the peculiarity of the theory of virtual particles and why s hould $\mathrm{i}$ t be us ed $\mathrm{t}$ o de scribe $\mathrm{c}$ ognitive processes in the human b rain? V irtual $p$ article (VP) is quanta of relativistic wave fields participating in vacuum fluctuations. From $t$ he general $q$ uantum-mechanical point of view, VP can be considered as particles arising in $\mathrm{i}$ ntermediate states o $\mathrm{f}$ transition $\mathrm{p}$ rocesses a nd interaction of $\mathrm{p}$ articles. VPs $\mathrm{h}$ ave th e $\mathrm{s}$ ame $\mathrm{q}$ uantum numbers as ordinary real particles, the quantum numbers of which, i nt urn, c orrespond t o o ne o f t he $\mathrm{r}$ eal elementary particles (with mass $\mathrm{m}$ ), for which, however, the usual connection between en ergy and momentum is not satisfied.

Which means that for them:

$$
E^{2} \neq m^{2} c^{4}+p^{2} c^{2}
$$

Where $\mathrm{c}$ is the speed of light in vacuum.
Therefore, a $\mathrm{m}$ easure of " virtuality" of the particle was introduced, which is expressed as:

$$
Q^{2}=E^{2}-m^{2} c^{4}-p^{2} c^{2}
$$

Virtual p articles c annot " travel in finitely"; t hey a re generated and must either be absorbed by some particle or $d$ isintegrate [14]. B asically, $v$ irtual $p$ articles a re the way the interaction takes place.

This is v ery c lose to the concept of I Rs for h uman interaction, $b$ ecause $t$ hey a re a lso $\mathrm{p}$ resent o nly i $\mathrm{n} t$ he virtual world, but also have material properties, can have a co ncrete i mpact on the matter ( on the human body), and are designed to describe the processes of exchange, generation, and processing of information by an individual. I Rs a lso c annot " exist in finitely"; $t$ heir existence is conditioned exclusively by the presence of a perceptive individual (imagine a $\mathrm{p}$ icture that no o ne is looking at). Therefore, each information image must also be absorbed or embodied in a material form [15].

Additionally, VPs a re $r$ esponsible for the qu antum mechanism of $\mathrm{i}$ nteraction of $\mathrm{p}$ articles - they a re $\mathrm{t}$ he carriers of i nteractions. F or e xample, the s cattering of charged particles due to electromagnetic interactions between them on quantum field representations is carried out through the exchange of virtual photons.

Virtual particles exist in intermediate short duration states, for which the usual $r$ atio b etween e nergy, $p$ ulse and mass i s $\mathrm{n}$ ot met. $\mathrm{O}$ ther ch aracteristics of virtual particles ar e el ectric ch arge, s pin, baryon ch arge, et c. are the same as for corresponding real particles [14].

The co ncept o f" Virtual $\mathrm{P}$ articles" an $\mathrm{dv}$ irtual processes is central to modern quantum field theory. In this theory, the interaction of particles and their mutual transformations ar e co nsidered as $t$ he $g$ eneration o $r$ absorption $b$ y o ne $f$ ree $p$ article o fo ther (virtual) particles. Any p article c ontinuously e mits and a bsorbs VPs of different types. For example, a proton emits and absorbs $v$ irtual pi -mesons ( and ot her VPs) a nd t hus appears surrounded by a cloud. In this case, the number of VPs is uncertain.

Here we can also draw an analogy with an individual who g enerates a " cloud" o f $t$ heir i nformation i mages, which essentially represent their mind and accumulated individual and social experience [15].

In addition, from the point of view of classical physics, a free particle (a particle which is not affected by e xternal forces, i .e. $r$ esting o $r$ moving e venly a nd linearly) can neither generate nor absorb another particle (for example, a free electron can neither emit nor absorb a photon), since such an occurrence would violate either the 1 aw of $\mathrm{c}$ onservation of e nergy or the la w o f conservation of momentum. Indeed, the resting el ectron has the minimum pos sible energy. That is why such an electron can not e mit a $p$ hoton $t$ hat al ways $p$ ossesses energy, a $s$ th is would violate th e la w o f e nergy conservation. If the electron moves at a constant speed, it also cannot (due to its kinetic energy) generate a photon, because this would $v$ iolate the $l$ aw of co nservation of momentum: the electron's momentum loss caused by the energy loss from photon generation would be larger than the i mpulse of the photon c orresponding to i ts e nergy 
(due to the difference in masses of these particles). The same applies to the process of absorption of the photon by a free electron.

However, the situation in quantum mechanics is quite different. According to the fundamental principle of quantum $m$ echanics - the uncertainty $p$ rinciple ( see Indeterminacy $R$ elation), $t$ he en ergy o f a ny $p$ article "existing" in a small interval of time $\Delta t$ is not fixed. The energy and momentum are continuously fluctuating, and during $\mathrm{s}$ mall i ntervals o $\mathrm{ft}$ ime, $\mathrm{t}$ he $\mathrm{l}$ aw o fe nergy conservation may b e " temporarily $\mathrm{v}$ iolated" ( in th $\mathrm{e}$ classical sense), while $p$ rocesses o ccurring at small scales may be acco mpanied by "local violations" of the law of conservation of momentum [14].

Moreover, it would $\mathrm{n}$ ot $\mathrm{b}$ e accu rate $\mathrm{t}$ o $\mathrm{r}$ efer $\mathrm{t} o$ conservation laws in the u sual s ense in the cas e of IR, which, of course, are limited to certain energy indicators based o $\mathrm{n} t$ heir material car riers, $\mathrm{i}$.e. - human $\mathrm{b}$ eings. However, if we ar e $\mathrm{n}$ ot $\mathrm{t}$ alking ab out $\mathrm{t}$ he en tire I R system, but about its individual information images, it is obvious that th e c lassical c onnection (1) will a lso b e broken ( in $\mathrm{p}$ revious models I Rs were $\mathrm{p}$ resented as a higher number of interacting particles [15]).

Sometimes, for the sake of clarity, the co ncept of "virtual particles" is explained somewhat differently. Namely, it is mentioned that in $\mathrm{t}$ he $\mathrm{p}$ rocess o $\mathrm{f}$ interaction, t he 1 aw of en ergy co nservation is s atisfied with some permissions. This does not contradict quantum mechanics: a ccording t o t he i ndeterminacy principle, an event lasting for a finite period of time does not al low to $d$ etermine $t$ he en ergy with a $n$ accu racy exceeding a cer tain 1 imit. $\mathrm{R}$ oughlys peaking, intermediate particles "borrow energy" for a s hort time. In $t$ his cas $e$, o rdinary $p$ articles ca $n b$ e generated an $d$ disappear in the process of interaction, only with a small violation of the law of energy conservation.

The interaction of ordinary, real particles in the vast majority of $\mathrm{c}$ ases oc curs $\mathrm{v}$ ia e mission a nd a bsorption (exchange) of VPs. T he e nergy a nd momentum of real particles before and after the reaction remain unchanged, and during the reaction, the laws of preservation of these values are not satisfied. The whole theory is constructed in such a way that any reaction can be represented as a result of various virtual $p$ rocesses o ccurring $d$ uring a short reaction time [16].

Additionally, the in teraction o $\mathrm{f} i$ ndividuals is a process of emission and absorption (processing and perception) o fI Rs. Thus, we can d escribe an $y$ information interaction a $\mathrm{s}$ a result of virtual $\mathrm{p}$ rocesses related to IR.

Thus, $t$ here ar e quite a $f$ ew an alogies b etween $t$ he processes of i nteraction of particles i $\mathrm{n} q$ uantum field theory and individuals in the theory of information images. Of course, such analogies can not be interpreted as a sign of a complete concordance of the two theories; however, $\mathrm{i} t \mathrm{~s}$ hould $\mathrm{b}$ e $\mathrm{t}$ aken $\mathrm{i}$ nto a ccount $\mathrm{t}$ hat $\mathrm{a} \mathrm{n}$ individual is also a part of nature, and, therefore, those patterns, 1 aws, $p$ henomena $t$ hat $s$ urround $u$ s ( not o nly phenomena r elated to VPs b ut a lso d iffusion, laws of conservation, et c.) must s omehow manifest $\mathrm{i} n$ individuals and their cognitive processes.
However, this can only be reliably verified by using the appropriate mathematical apparatus to predict specific co gnitive $p$ rocesses an $d v$ erify $r$ esults $i \mathrm{n}$ a $n$ experimental study.

\section{Mathematical apparatus}

The classical method for describing a $\mathrm{v}$ irtual process ( $\mathrm{a}$ process in volving $\mathrm{v}$ irtual $\mathrm{p}$ articles) is $\mathrm{t}$ he $\mathrm{F}$ eynman diagram method [17]. However, the direct application of such a device for IRs would hardly be possible. First, it is $n$ ecessary $t o p$ erform $p$ arameterization o f I Rs a nd compare these values with VP parameters.

Interactions of virtual $\mathrm{p}$ articles ar e $\mathrm{d}$ escribed $\mathrm{b}$ y $\mathrm{t}$ he propagator ( distribution $f$ unction) - a $f$ unction th at determines the amplitude of probability of distribution of a $r$ elativistic $f$ ield ( particle) $b$ etween $t$ wo $s$ tages o $f$ interaction. The pr opagator is de termined $t$ hrough the evolutionary operator:

$$
D\left(x, t ; x^{\prime}, t^{\prime}\right)=\left\langle x\left|\widehat{U}\left(t, t^{\prime}\right)\right| x^{\prime}\right\rangle
$$

It allows considering the influence of $\mathrm{v}$ irtual particles. In $\mathrm{f}$ act, the propagator is a function o $\mathrm{ft}$ he Green's wave eq uation. I ng eneral, i n el ementary particle physics and statistical physics, Green's functions are often used as propagators in Feynman diagrams (and the e xpression " Green's function" i s of ten a pplied i $\mathrm{n}$ general to $\mathrm{t}$ he $\mathrm{c}$ orrelation $\mathrm{f}$ unction in $\mathrm{q}$ uantum field theory).

Within the model, the interaction of two individuals is represented as the interaction of two systems with the help o f co mmunication fields ( which ar e q uantized through I R - as a $n$ a nalog of V P) i $n$ the information environment. Also, o fi nterest i s t he i mpact o n o ne individual o f a given i nformation e nvironment ( e.g., media, Internet resources, social environment, etc.).

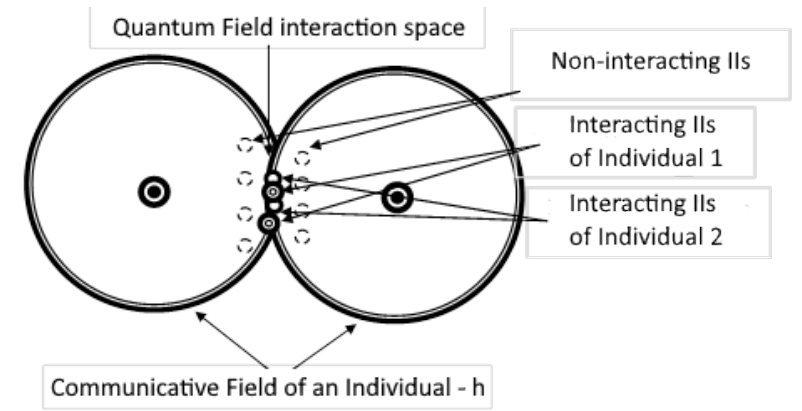

Fig. 1. Interaction of communicative fields of two individuals

The interaction function for these two individuals or an individual and the external a ction should be written accordingly.

Since we are talking about the IR space, it is obvious that this space will have certain coordinates; the particles simulating the IR movement in this space will also have a cer tain momentum, e nergy. At $t$ he $s$ ame $t$ ime, we should $\mathrm{k}$ eep i $\mathrm{n}$ mind $\mathrm{t}$ he $\mathrm{p}$ resence o f 1 ocal minimum potential particle energy in the space. Thus, an individual's mind can be represented as a potential well, 
inside of which information representations $\mathrm{m}$ ake vibrational movements. It is clear, that it is common for physics to solve the problem of quantization of the field inside the potential well to determine energy levels (by solving t he stationary S chrödinger e quation [ 18]). However, in this cas e, we know ab out the presence of particles in advance.

The potential well can be represented in the simplest form as - Fig.2.

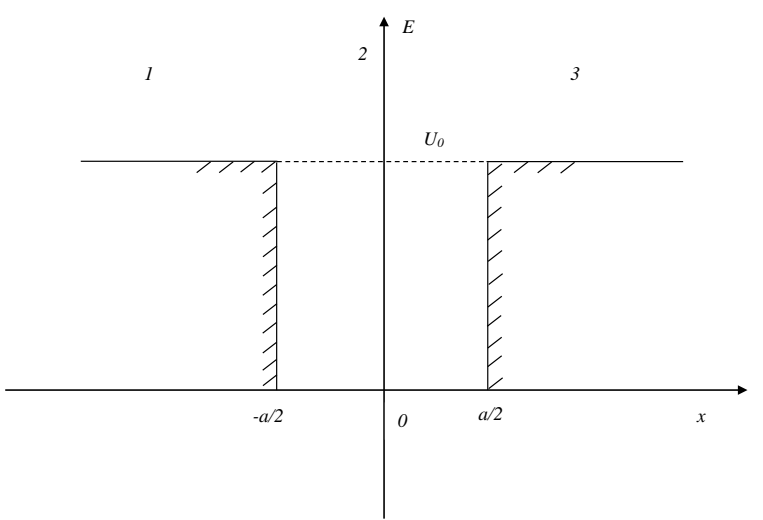

Fig. 2. The most basic one-dimensional potential well with finite walls.

$$
U(x)=\left\{\begin{array}{c}
0,-\frac{a}{2}<x<\frac{a}{2}, \text { region } 2 \\
x \geq \frac{a}{2}, \text { region } 3 \\
U_{0}, \quad x-\frac{a}{2}, \text { region } 1
\end{array}\right.
$$

When a $p$ article crosses the potential $b$ arrier in the form of a wall, it simulates the information interaction of an in dividual (i.e., $p$ article $d$ isturbance, $t$ he tr ansition from one energy level to a higher one).

A more interesting case of a potential well is the well uneven walls a nd a $\mathrm{n}$ a dditional i nternal $\mathrm{b}$ arrier. S uch barrier an $\mathrm{d} h$ eterogeneity o $\mathrm{f}$ walls s imulate cer tain specific properties of the human mind, in particular, the conditional d ivision in to $\mathrm{c}$ onsciousnesssubconsciousness, complexity of interaction with the external e nvironment, e tc. T heoretically, de pending on the mental $\mathrm{s}$ tate o $\mathrm{f}$ th $\mathrm{e}$ modeled in dividual ( or th eir individual cognitive function), such barriers may be more complex and repeated multiple times - Fig.3.

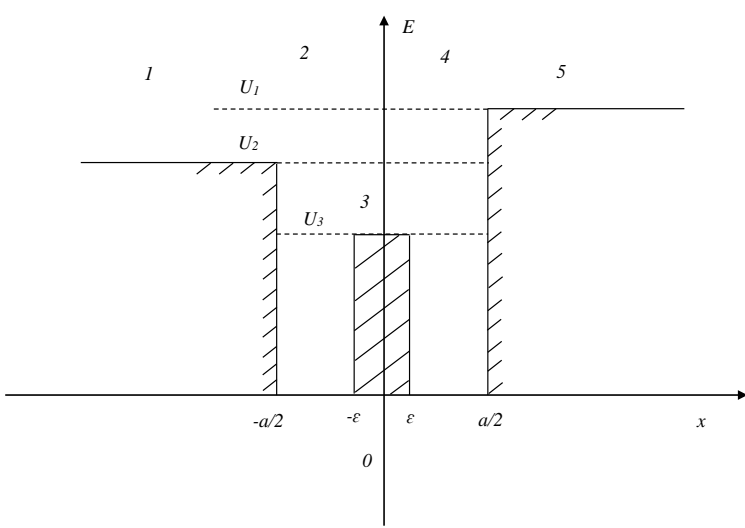

Fig .3. One-dimensional potential well with finite walls. A version with uneven walls and internal potential barrier.

$$
U(x)=\left\{\begin{array}{c}
U_{1}, x \geq \frac{a}{2} \\
0, \varepsilon<x<\frac{a}{2} \\
U_{2},-\varepsilon \leq x<\leq \varepsilon \\
0,-\frac{a}{2}<x<-\varepsilon \\
U_{0}, x \leq-\frac{a}{2}
\end{array}\right.
$$

Thus, $\mathrm{t}$ he impact of $\mathrm{t}$ he external information environment o $\mathrm{nt}$ he mind o fa $\mathrm{ni}$ ndividual $\mathrm{c}$ an $\mathrm{b} e$ described as $t$ he $i$ mpact $o n p$ articles ( modeling information images) in a potential well. Let us record the equations for the case in Fig. 2.

The Schrödinger equation outside the potential well for the information image (IR) will look as:

$$
\frac{\mathrm{d}^{2} \varphi_{\text {out }}(\mathrm{x})}{\mathrm{dx}^{2}}-\frac{2 \mathrm{~m}}{\hbar^{2}}\left(\mathrm{U}_{0}-\mathrm{E}\right) \varphi_{\text {out }}=0
$$

Where,

$\varphi_{\text {out }}$ is a wave function outside the potential well.

$E$ is the total energy of an IR.

$m$ is mass (complexity) of IR.

$\hbar$ is Planck constant.

By introducing the interaction coefficient $k_{1}$,

$$
k_{1}=\sqrt{\frac{2 m}{\hbar^{2}}\left(U_{0}-E\right)}
$$

We obtain,

$$
\frac{d^{2} \varphi_{\text {out }}(x)}{d x^{2}}-k_{1}^{2} \varphi_{\text {out }}=0
$$

For region 1, the solutions of this equation will look as:

$$
\begin{aligned}
& \varphi_{1}(\mathrm{x})=\mathrm{A}_{1} \mathrm{e}^{\mathrm{k}_{1} \mathrm{x}}+\mathrm{B}_{1} \mathrm{e}^{-\mathrm{k}_{1} \mathrm{x}} \\
& \varphi_{3}(\mathrm{x})=\mathrm{A}_{3} \mathrm{e}^{\mathrm{k}_{1} \mathrm{x}}+\mathrm{B}_{3} \mathrm{e}^{-\mathrm{k}_{1} \mathrm{x}}
\end{aligned}
$$

For th e wave function to be li mited ( which is impossible otherwise for real information images), $B_{1}$ и $A_{3}=0$.

For r egion 2 , lo cated in the well (i.e. in the human mind), it will look as:

$$
\frac{\mathrm{d}^{2} \varphi_{\text {out }}(\mathrm{x})}{\mathrm{dx}^{2}}+\frac{2 \mathrm{~m}}{\hbar^{2}}(\mathrm{E}) \varphi_{\text {out }}=0
$$

Here, the interaction coefficient $k_{2}$ will be,

$$
\mathrm{k}_{2}=\sqrt{\frac{2 \mathrm{~m}}{\hbar^{2}} \mathrm{E}}
$$

And the solution will look as:

$$
\varphi_{2}(\mathrm{x})=\mathrm{C} \sin \left(\mathrm{k}_{2} \mathrm{x}+\propto\right)
$$


Thus, the wave functions for an information image in the mind of an individual will have a form:

$$
\begin{gathered}
\varphi_{1}(\mathrm{x})=\mathrm{A}_{1} \mathrm{e}^{\mathrm{k}_{1} \mathrm{x}} \\
\varphi_{2}(\mathrm{x})=\mathrm{C} \sin \left(\mathrm{k}_{2} \mathrm{x}+\propto\right) \\
\varphi_{3}(\mathrm{x})=\mathrm{B}_{3} \mathrm{e}^{-\mathrm{k}_{1} \mathrm{x}}
\end{gathered}
$$

The ex ternal impact will be recorded by disturbance $\mathrm{V}(\mathrm{x})$, which i s a dded $\mathrm{t}$ ot he ge neral form o $\mathrm{ft}$ he Schrödinger equation:

$$
\frac{d^{2} \varphi(x)}{d x^{2}}-\frac{2 m}{\hbar^{2}}(U(x)+V(x)-E) \varphi=0
$$

Disturbance can be set in different ways, depending on its type, for example, by normal distribution or other methods.

This model s hould be come $\mathrm{t}$ he foundation for experimental $r$ esearch a nd $p$ ractical $t$ esting o $\mathrm{ft}$ he modeling results in the future.

\section{Conclusion}

Thus, in this paper, we presented the fundamentals of the mathematical model of $\mathrm{t}$ he human mind $\mathrm{b}$ ased o $\mathrm{n} t$ he mathematical ap paratus of $q$ uantum mechanics (primarily, $t$ he $\mathrm{p}$ otential wells a nd e lements of virtual particles in the problem statement), d efined the task of modeling the cognitive activity of the human brain based on cl assical mathematical models an $\mathrm{dp}$ hysical analogies, $g$ ave $a b$ rief o verview o $f t$ he $t$ heory o $f$ information $r$ epresentations a nd $i$ ts foundations, highlighted the general $r$ egularities and the $p$ henomena connected with the behavior of information representations of the human mind and virtual particles and proposed options of pot ential wells f or modeling cognitive brain functions.

This allowed us $\mathrm{t}$ o pr opose a f oundation for a mathematical model, which will b ef urther te sted experimentally (based on external information impact on the individual and his psychophysiological reactions).

The results of $\mathrm{t}$ hese $\mathrm{s}$ tudies can $\mathrm{b}$ e $\mathrm{u}$ sed $\mathrm{t} \mathrm{o}$ cr eate predictive models of $\mathrm{V}$ arious $\mathrm{c}$ ognitive di sorders (diseases) and to be used in diagnostics. First of all, this concerns the problems of perception of information, the processing o f co mplex i mages ( connecting $d$ ifferent categories of information, for example, color and sound), etc.

\section{References}

1. Y.I. Aleksandrov, Proceedings of ISA RAS, 61(3), 3-25 (2011)

2. D.S.C hernavsky, Synergy and information. Dynamic information theory. (URSS, 2009)

3. W. Gevers, R.C. Kadosh, W. Notebaert, Journal of Experimental Psychology: Learning, Memory, and Cognition, 37(5), $1243 \quad-1249 \quad$ (2011) DOI:10.1037/a0023550
4. T.M. Lee, H.L. Liu, C.C. Chan, (Eds.), Neuroimage. 28(2), $305 \quad-313 \quad$ (2005) https://doi.org/10.1016/j.neuroimage.2005.06.051

5. D. Griffith, F. G reitzer, Cognitive informatics and natural i ntelligence, 1(1), $3 \quad 9-52 \quad$ (2007) DOI:10.4018/jcini.2007010103

6. J. Vandekerckhove, Journal o fM athematical Psychology, 60, 58-71 (2014)

7. O. Faugeras, J. I nglis, Journal o f $\mathrm{M}$ athematical Biology, 71(2), 259-300 (2015)

8. B.W. Kooi, J. M ath. B iol. 71, 1575-1605 (2015) DOI: $10.1007 / \mathrm{s} 00285-015-0869-0$

9. P. Haazebroek, S . van D antzig, B . Hommel, Cognitive $\mathrm{P}$ rocessing, 12(4), 3 55-365 ( 2011) DOI:10.1007/s10339-011-0408-x

10. B.B. Velichkovsky, A .N. Gusev, V.F. Vinogradova, O .A. Arbekova, Experimental psychology, 9(1), 5-20 (2016) [in R ussian] DOI:10.17759/exppsy.2016090102

11. K.V. Anokhin. Probes for Mapping Nervous Networks during Training. Principles and mechanisms of human brain activity (L.: $\mathrm{N}$ auka, 1989)

12. F. Pana, et al, Neuroscience L etters, 628, 35-39 (2016) https://doi.org/10.1016/j.neulet.2016.05.062

13. A.R. N ikolaev, R .N. Meghanathan, C. van Leeuwen, Brain and Cognition, 107, 55-83 (2016) https://doi.org/10.1016/j.bandc.2016.06.004

14. Microcosm Physics (1980)

15. A.Y. Petukhov, S.A. Polevaya, International Journal of B iomathematics, 10(6), 1750092 (2017) DOI:10.1142/S1793524517500929

16. C. Wilson, G. Johansson, A . Pourkabirian, et al, Nature, 479, $37 \quad 6-379$ ( 2011) https://doi.org/10.1038/nature10561

17. R. Feynman, Theory of Fundamental Processes (M., 1978)

18. A. Bohm, Quantum mechanics: foundations and applications (M. Mir, 1990) 